\title{
Analytical tools for the nanoworld
}

\section{Renato Zenobi}

Published online: 13 November 2009

(C) Springer-Verlag 2009

The theme of nanoscale chemical analysis is gaining importance and momentum. Modern nanotechnology is capable of fabricating a large variety of nanoscale objects, elements for molecular electronics, nanoparticles, and nanoscale surface structures. For the detailed characterization of such objects, but also of subcellular compartments, 'molecular machines', and other very small biological structures, there is currently a lack of methods for chemical diagnostics and characterization, in particular of the molecular composition. The challenge arises because traditional methods with nanoscale lateral resolution (e.g. standard atomic force microscopy, scanning tunnelling microscopy, and electron microscopy) typically yield very little or no chemical information, whereas traditional methods of chemical analysis (e.g. NMR, mass spectrometry, and even spectrochemical analyses carried out with microscopes) are in many cases very far from achieving nanoscale lateral resolution.

This special issue aims at presenting the state of the art in nanoscale chemical analysis. Fifteen contributionseight reviews and seven original research papers-from university institutes and research laboratories worldwide discuss how methods as diverse as electron microscopy, nano-optical methods, focused ion beams, specialized atomic force microscopy and scanning tunnelling microscopy methods, and nanoscale laser ablation mass spectrometry can provide answers to scientific questions ranging from electronic transport phenomena in nanostructures to inorganic nanocrystals, to single biological molecules and their interactions. I resist the temptation as guest editor of enumerating the directions and contents of these papers and warmly invite you to open the issue and have a look for yourself!

I would like to thank all authors for their contributions, and the Analytical and Bioanalytical Chemistry editors for the pleasant collaboration in putting this issue together.

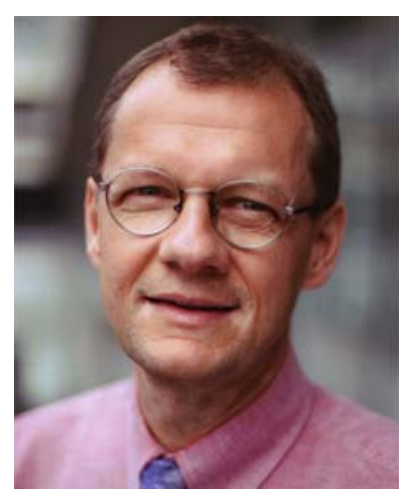

Renato Zenobi

has been Professor of Analytical Chemistry in the Organic Chemistry Laboratory of ETH Zurich since 1995. He received an M.S. degree from ETH Zurich in 1986 and a Ph.D. degree from Stanford University in the USA in 1990. He had two postdoctoral appointments at the University of Pittsburgh (1990-1991) and at the University of Michigan (1991). Renato Zenobi's research interests include laser-based analytical chemistry, electrospray and laserassisted mass spectrometry, laser-surface interactions, and near-field optical microscopy and spectroscopy. He has received numerous awards for his scientific work, including the Thomas Hirschfeld Award, the Ruzicka Prize, the Heinrich Emanuel Merck Prize, the Theophilus Redwood Lectureship, and the Michael Widmer Award.

R. Zenobi $(\bowtie)$

Department of Chemistry and Applied Biosciences,

HCI E 329, ETH Zurich,

8093 Zurich, Switzerland

e-mail: renato.zenobi@org.chem.ethz.ch 